\title{
When and where did GW150914 form?
}

\author{
A. Lamberts, ${ }^{1 \star}$ S. Garrison-Kimmel, ${ }^{1 \star}$ D. R. Clausen ${ }^{1,2}$ and P. F. Hopkins ${ }^{1,2}$ \\ ${ }^{1}$ TAPIR, MC 350-17, California Institute of Technology, Pasadena, CA 91125, USA \\ ${ }^{2}$ Walter Burke Institute for Theoretical Physics, MC 452-48, California Institute of Technology, Pasadena, CA 91125, USA
}

Accepted 2016 July 27. Received 2016 July 13; in original form 2016 May 27

\begin{abstract}
The recent LIGO detection of gravitational waves (GW150914), likely originating from the merger of two $\sim 30 \mathrm{M}_{\odot}$ black holes suggests progenitor stars of low metallicity $\left(\left[Z / \mathrm{Z}_{\odot}\right] \lesssim\right.$ $0.3)$, constraining when and where the progenitor of GW150914 may have formed. We combine estimates of galaxy properties (star-forming gas metallicity, star formation rate and merger rate) across cosmic time to predict the low redshift black hole - black hole merger rate as a function of present day host galaxy mass, $M_{\mathrm{gal}}$, the formation redshift of the progenitor system $z_{\mathrm{f}}$ and different progenitor metallicities $Z_{\mathrm{p}}$. For $Z_{\mathrm{p}} \geqslant 0.1 \mathrm{Z}_{\odot}$, the signal is dominated by binaries in massive galaxies with $z_{\mathrm{f}} \simeq 2$ while below $Z_{\mathrm{p}} \leqslant 0.1 \mathrm{Z}_{\odot}$ most mergers come from binaries formed around $z_{\mathrm{f}} \simeq 0.5$ in dwarf galaxies. Additional gravitational wave detections from merging massive black holes will provide constraints on the mass-metallicity relation and massive star formation at high redshifts.
\end{abstract}

Key words: gravitational waves-binaries: close-stars: black holes-stars: evolutiongalaxies: abundances-galaxies: stellar content.

\section{INTRODUCTION}

On 2015 September 14, both detectors from the Laser Interferometer Gravitational-Wave Observatory (LIGO) made the first direct detection of gravitational waves (Abbott et al. 2016b). The gravitational waves were emitted by two merging black holes of $M_{1}=36_{-4}^{+5} \mathrm{M}_{\odot}$ and $M_{2}=29_{-4}^{+4} \mathrm{M}_{\odot}$ located at redshift $z=0.09_{-0.04}^{+0.03}$. While the detection of black holes much heavier than any mass measured in X-ray binaries revives the study of the evolution of massive stellar binaries (Mandel \& de Mink 2016; Marchant et al. 2016), the determination of host galaxies has been mostly ignored. So far, binary population syntheses (BPS) models have argued that GW150914 can only have formed in a low-metallicity environment, below $0.25 \mathrm{Z}_{\odot}$, most probably around $0.1 \mathrm{Z}_{\odot}$ (Abbott et al. 2016d; Belczynski et al. 2016).

This strong limit on the progenitor metallicity allows one to determine in what type of galaxy and at what time the progenitors of massive $\left(M_{1}+M_{2} \geqslant 40 \mathrm{M}_{\odot}, M_{1}, M_{2} \geqslant 15 \mathrm{M}_{\odot}\right)$ binary black holes $(\mathrm{BBH})$ are born. While previous work has determined merger rates as a function of redshift (Dominik et al. 2013; Dvorkin et al. 2016), this Letter presents the first determination of the formation conditions for the massive BBH mergers we currently observe. As the delay time between progenitor formation and BBH merger often exceeds several Gyr, one has to consider star formation through cosmic history to correctly model the progenitor population.

Low-metallicity gas is typically found in high-redshift galaxies or in local dwarf galaxies. Using a two-component model for the star formation and metallicity as a function of redshift, O'Shaughnessy, Kalogera \& Belczynski (2010) showed that elliptical galaxies dominate $\mathrm{BBH}$ mergers hosts. Based on the redshift evolution of a mass-independent metallicity distribution with significant scatter, Belczynski et al. (2016) suggests two roughly equally probable formation times for GW150914 around $z \simeq 3$ and below $z \simeq 0.2$. In this Letter, we use a complete, redshift dependent mass-metallicity relation (MZR) consistent with recent high-redshift observations (Erb et al. 2006; Mannucci et al. 2009). Additionally, we explicitly account for galaxy mergers that bring low-metallicity stars/black holes formed in low-mass galaxies to higher mass galaxies, where the BBH mergers take place.

In order to determine the environment in which GW150914 formed, we assume the progenitors have the metallicity of the gas in which they form. First, we determine the amount of low-metallicity star formation through cosmic history (Section 2). Using a BPS model, we then determine the delay time distribution for various progenitor metallicities (Section 3). We finally combine both computations to determine where GW150914 most likely formed (Section 4) and discuss the implications for future detections (Section 5). In this Letter, we assume a $\Lambda$ Cold Dark Matter cosmology with $h=0.7, \Omega_{\Lambda}=0.7$ and $\Omega_{\mathrm{m}}=0.3$ and use $\mathrm{Z}_{\odot}=0.02$.

\section{FORMING LOW-METALLICITY STARS}

The total merger rate $\mathcal{R}$ at merger time $t_{\mathrm{m}}$ is given by

$$
\begin{aligned}
\frac{\mathrm{d} \mathcal{R}}{\mathrm{d} \log M_{\mathrm{gal}} \mathrm{d} t_{\mathrm{f}} d Z_{\mathrm{p}}}= & \frac{\mathrm{d} N}{\mathrm{~d} \log M_{\text {gal }} d Z_{\mathrm{p}}} \int_{t_{\infty}}^{t_{\mathrm{m}}} \mathrm{d} t_{\mathrm{f}} \frac{\mathrm{d} N_{\mathrm{m}}\left(t_{m}-t_{\mathrm{f}}, Z_{\mathrm{p}}\right)}{\mathrm{d} t_{f}} \\
& \operatorname{SFR}\left(t_{\mathrm{f}}, M_{\mathrm{gal}}\right) \Psi\left(t_{\mathrm{f}}, M_{\mathrm{gal}}, Z_{\mathrm{p}}\right),
\end{aligned}
$$




\section{L32 A. Lamberts et al.}

where $M_{\mathrm{gal}}$ is the galaxy stellar mass, $Z_{\mathrm{p}}$ the progenitor metallicity. We perform the integral over the formation time of the progenitors $t_{\mathrm{f}}$. SFR is the star formation rate (in $M_{\odot} \mathrm{yr}^{-1}$ ) and $\mathrm{d} N / \mathrm{d} \log M_{\mathrm{gal}}$ the stellar mass function (SMF) at the time of the merger [per unit comoving volume, taken from Tomczak et al. (2014)]. $\Psi$ is the fraction of stellar mass forming at metallicity $Z_{\mathrm{p}}$ with respect to the total stellar mass formed. $\mathrm{d} N_{\mathrm{m}} / \mathrm{d} t$ is the delay time distribution of massive black hole mergers per unit solar mass. In this section, we will determine the distribution of low-metallicity stellar mass as a function of host mass and formation time while in Section 3, we will determine the number of black hole mergers per unit solar mass $N_{\mathrm{m}}$.

To determine the amount of low-metallicity stellar mass in each galaxy, we include stars formed within that galaxy as well as stars that were brought into the galaxy through mergers. While this 'ex situ' star formation is around 30 percent in present day galaxies (Lackner et al. 2012), the accreted stars are typically formed in lower mass galaxies, which have a lower metallicity. As such, the ex situ component cannot be neglected for our Letter. The study of galaxy merger histories can be done with hydrodynamic cosmological simulations (e.g. Maller et al. 2006) or semi-analytic models of galaxy formation within dark matter haloes (e.g. Cole et al. 2000; Fakhouri \& Ma 2008; Guo \& White 2008). We use the global fit function from Cole et al. (2008), based on the extended Press-Schechter formalism (Lacey \& Cole 1993), which provides the redshift dependent mass distribution of progenitor haloes that will merge within the main halo by $z=0$. We neglect the evolution of the main halo and galaxy mass between $z_{\mathrm{m}}$ and $z=0$, but for the observed $z_{\mathrm{m}}$ this is extremely small.

For each galaxy mass we consider, we determine the mass of the corresponding dark matter halo using abundance matching by Behroozi, Wechsler \& Conroy (2013). Using 10 Myr timesteps, we build up the amount of low-metallicity stars within that halo between $z_{\infty}=8$ and $z_{\mathrm{m}}$ according to the merger tree. We neglect stars formed before $z=8$ due to the lack of observational constraints. While the SFR density at such early times is at least two orders of magnitude below its value at the peak of star formation the lowmetallicity environment will increase their respective contribution to the total merger rate. For each timestep, we determine the progenitor halo mass function. For each of the progenitors, we determine the corresponding galaxy mass and SFR at that redshift, again using data from Behroozi et al. (2013). We then determine the fraction of stars forming at $Z_{\mathrm{p}}$ using the redshift dependent MZR. Finally, we add up the low-metallicity contributions of all the progenitors to get the total amount of low-metallicity stars formed at the considered redshift that will be in $M_{\mathrm{gal}}$ at $z_{\mathrm{m}}$.

We model 11 metallicity bins between $Z_{\mathrm{p}}=0.01 \mathrm{Z}_{\odot}$ and $Z_{\mathrm{p}}=Z_{\odot}$, each bin being 0.2 dex wide. We specifically examine $\mathrm{BBH}$ progenitors formed at $Z_{\mathrm{p}} / \mathrm{Z}_{\odot}=0.3,0.1$, and 0.01 ; $Z_{\mathrm{p}}=0.1 \mathrm{Z}_{\odot}$ is broadly the most likely value (Belczynski et al. 2016), but progenitors form for $Z_{\mathrm{p}} \lesssim 0.5 Z_{\odot}$. The observational determination of gas-phase metallicities, which is needed to tell us where low-metallicity stars form, unfortunately, has systematic uncertainties of $\simeq 0.5$ dex owing to different nebular calibrations (Kewley \& Ellison 2008; Steidel et al. 2014).

We therefore determine the mean metallicity of the star-forming gas using the mass-metallicity relation from Ma et al. (2016)

$12+\log (\mathrm{O} / \mathrm{H})=0.35\left(\log \left(M_{\mathrm{gal}}\right)-10\right)+0.93 \exp ^{-0.43 z}+7.95 .(2)$

This MZR is based on high-resolution cosmological zoom-in simulations suite FIRE (Hopkins et al. 2014), which reproduce the observed stellar mass-halo mass relation, Kennicutt-Schmidt

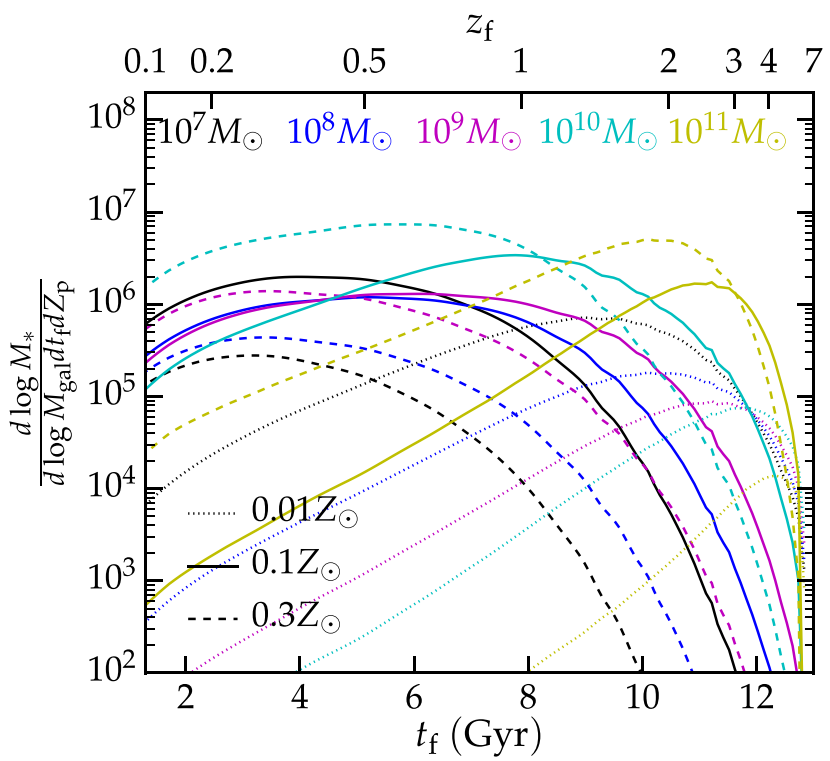

Figure 1. Cosmic mass density (in $\mathrm{M}_{\odot} \mathrm{Mpc}^{-3} \mathrm{Gyr}^{-1} \mathrm{M}_{\odot}^{-1}$ ) of stars at different metallicities (linestyles) in present-day galaxies with a total galaxy stellar mass $M_{\text {gal }}=10^{7-11} \mathrm{M}_{\odot}$ (colour as labelled), as a function of lookback time (redshift) to when the stars actually formed.

law, star-forming main sequence and star formation histories. More importantly, the simulated MZR agrees with both gas phase and stellar metallicity measurements observed at low redshifts for $10^{4} \leqslant M_{\text {gal }} \leqslant 10^{11} \mathrm{M}_{\odot}$ (Tremonti et al. 2004; Lee et al. 2006) as well as the data at higher redshifts (Erb et al. 2006; Mannucci et al. 2009). This MZR agrees well with the Pettini \& Pagel (2004) calibration, removing some of the systematic uncertainties. If, however, we systematically increase all metallicities by switching to the Kobulnicky \& Kewley (2004) calibration, we obtain the same relative merger rates but lower the total rate by a factor of 5 .

To determine the actual amount of low-metallicity star-forming gas within a galaxy, we need to assess the scatter with respect to the mean metallicity, as increased scatter will increase the number of BBH progenitors. Tremonti et al. (2004) indicate a scatter with $\sigma \simeq 0.1$ dex between different galaxies independent of redshift. This is significantly lower than the scatter derived from damped Ly $\alpha$ systems (DLA; Rafelski et al. 2012). In the latter, galaxy masses are not measured, and their scatter likely accounts for most of the scatter in metallicity (Dvorkin et al. 2015). A significant scatter may also be present within a given galaxy. In spiral galaxies, the metallicity decreases by about $0.03-0.06 \mathrm{dex} \mathrm{kpc}^{-1}$ with galactocentric radius (Henry et al. 2010; Berg et al. 2013). At a given radius, scatter is typically $\simeq 0.1 \mathrm{dex}$. Assuming both radial and non-radial variations of $\sigma \simeq 0.2$, we have a total standard deviation of $\sigma=0.3$. Using a normal distribution for $[\mathrm{O} / \mathrm{H}]$, we then determine $\Psi$, the fraction of gas at $Z_{\mathrm{p}}$.

Fig. 1 shows the low-metallicity stellar mass density as a function of lookback time (and redshift) to its formation for various galaxy masses (taken at $\left.z_{\mathrm{m}}=0\right) .{ }^{1}$ Stars with $Z_{\mathrm{p}}=0.01 \mathrm{Z}_{\odot}($ dotted lines) form before $z \simeq 2$ and can be found in dwarf galaxies. Stars with $Z_{\mathrm{p}}=0.3 Z_{\odot}$ (dashed lines) formed more recently $\left(1 \leq z_{\mathrm{f}}\right.$ $\leq 2$ ) in Milky Way type galaxies. Stars with $Z_{\mathrm{p}}=0.1 \mathrm{Z}_{\odot}$ show

\footnotetext{
${ }^{1}$ The data for Fig. 1 can be found at https://astridlamberts.wordpress.com/ datacodes/.
} 
a combination of both trends. When we neglect galaxy mergers, low-metallicity star formation is reduced by at least an order of magnitude and limited to $z_{\mathrm{f}} \geq 2$, with little dependence on $M_{\text {gal }}$. We find that most of the metal-poor stars formed at low redshifts were brought in through mergers and were formed in galaxies smaller that their final host. In the next section, we will determine the typical time between progenitor star formation and $\mathrm{BBH}$ merger in order to determine from which of these environments GW150914 most likely originated.

\section{TIME DELAYS FOR MASSIVE BBH MERGERS}

To link the SFR, progenitor metallicity, and host mass evolution discussed above with BBH mergers that are detectable by LIGO, we compute a set of BPS models. Many phases in the evolution of binary stars remain poorly understood and previous BPS studies have shown that this results in large uncertainties in the BBH merger rate (e.g. Lipunov, Postnov \& Prokhorov 1997; Sipior \& Sigurdsson 2002; Dominik et al. 2013). Since this Letter focuses on host galaxies, and not binary evolution, we consider a simple, single set of standard assumptions consistent with observational constraints. We note that our models do not include the recently proposed massive overcontact binary BBH formation channel (Mandel \& de Mink 2016; Marchant et al. 2016). We focus on field binaries and neglect BBHs that are dynamically formed in globular clusters (e.g. Downing et al. 2011; Rodriguez et al. 2015), which would typically form at high redshifts and preferentially reside in more massive galaxies. Lacking observational constraints, we also neglect $\mathrm{BBH}$ stemming from Pop III stars (Kinugawa et al. 2014), which are not likely candidates for GW 150914 (Hartwig et al. 2016) and which contribution to the gravitational wave background is still uncertain (Dvorkin et al. 2016).

The BPS models are computed with the binary star evolution code BSE described in Hurley, Tout \& Pols (2002), which we have updated to improve the treatment of massive binaries. We use the weaker, metallicity-dependent wind mass-loss prescriptions from Belczynski et al. (2010). Updated remnant mass prescriptions are taken from Belczynski et al. (2008). BH birth kicks are modelled following Dominik et al. (2013). This results in the production of BBHs with component masses $\gtrsim 25 \mathrm{M}_{\odot}$ that are not disrupted by powerful natal kicks. The kicks are drawn from a Maxwellian distribution of width $265 \mathrm{~km} \mathrm{~s}^{-1}$, reduced according to the amount of material that falls back after core collapse.

We have also updated the treatment of some mass transfer scenarios in BSE. We force systems that experience a common envelope phase while the mass donor is in the Hertzsprung gap to merge. ${ }^{2}$ For stars that have evolved beyond the Hertzsprung gap, we take the common envelope efficiency to be unity, and compute the envelope binding energies with the BSE-default, evolutionary-state-dependent formulae. Furthermore, we allow stable Roche lobe overflow mass transfer to be non-conservative and assume that only half of the mass lost by the donor is accreted by the companion (Dominik et al. 2013). With this updated version of BSE we are able to produce a reasonable estimate for the $\mathrm{BBH}$ merger delay time distribution given an initial population of binary stars.

\footnotetext{
${ }^{2}$ Stars in the Hertzsprung gap lack a steep density gradient between the core and envelope so there is no clear boundary to halt the inspiral of the companion and prevent a stellar merger (Ivanova \& Taam 2004; Belczynski et al. 2007).
}

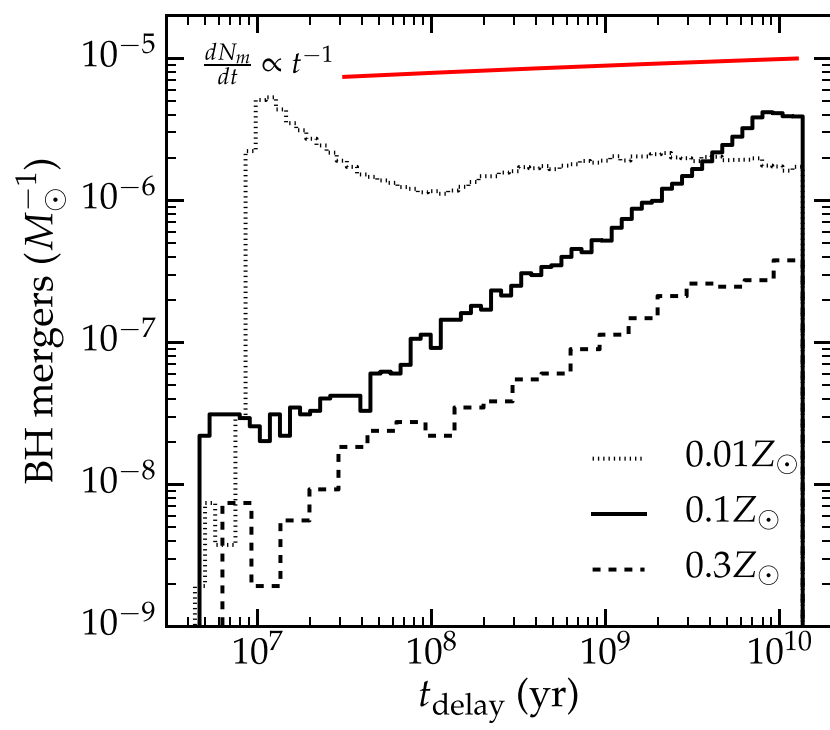

Figure 2. Number of massive BBH mergers per solar mass of star formation $N_{\mathrm{m}}$ as a function of time since formation for a stellar population with a Kroupa IMF and BBH mass $>40 M_{\odot}$. The upper limit in $t_{\text {delay }}=t_{\mathrm{m}}-t_{\mathrm{f}}$ is the Hubble time. For massive $\mathrm{BBH}$ mergers, only the $0.01 \mathrm{Z}_{\odot}$ population follows the standard $\mathrm{d} N_{\mathrm{m}} / \mathrm{d} t \propto t^{-1}$ evolution, shown with a red line.

We construct the delay time distribution from a Monte Carlo ensemble of $2.5 \times 10^{6}$ binaries. Primary masses range from 25 to $150 \mathrm{M}_{\odot}$ and are drawn from the initial mass function (IMF) given by Kroupa (2001). This allows for a wider mass distribution than the GW150914 event, which will be representative for future massive black hole binary detections. When we select a narrow mass range, set by the uncertainties on the GW150914 detection $\left(M_{1}=36_{-4}^{+5} \mathrm{M}_{\odot}\right.$ and $\left.M_{2}=29_{-4}^{+4} \mathrm{M}_{\odot}\right)$, we find qualitatively very similar trends. The initial mass ratios and orbital periods are drawn from the distributions measured by Sana et al. (2012). Initial eccentricities are drawn from a thermal distribution $f(e) \propto 2 e$. We evolve the same population of binaries for the 11 metallicity bins we consider.

Fig. 2 shows the number of BBH mergers per solar mass of stars formed that occur at time $t_{\text {delay }}$ after the stellar binary forms. We only considered BBH mergers with total mass larger than $40 \mathrm{M}_{\odot}$. Due to the metallicity dependence of the wind mass-loss rates, binaries formed at $Z_{\mathrm{p}}=0.01 \mathrm{Z}_{\odot}$ produce the most massive BHs. Accordingly, these extremely low-metallicity stars have the largest number of massive BBH mergers per unit stellar mass. However, at very late times higher metallicity stars account for a comparable number of mergers.

If we include $\mathrm{BBH}$ mergers of all masses (not shown here), $\mathrm{d} N_{\mathrm{m}} / \mathrm{d} t$ at each metallicity considered here approaches the standard $t^{-1}$ dependence (e.g. Dominik et al. 2013; Belczynski et al. 2016). This agreement with previous work is encouraging because, for our purposes, it is most important to properly capture the shape of the delay time distributions. When we restrict our study to BBH mergers with total mass larger than $40 \mathrm{M}_{\odot}$, only the $Z_{\mathrm{p}}=0.01 \mathrm{Z}_{\odot}$ delay time distribution $\mathrm{d} N_{\mathrm{m}} / \mathrm{d} t$ follows the $t^{-1}$ dependence, as is shown by the flat line for $N_{\mathrm{m}}(t)$. At higher metallicity, short mergers are absent because of larger stellar radii, which make many systems merge as stellar binaries before producing a $\mathrm{BBH}$. On top of that, some binaries contract less during the common envelope phase, because of the lower envelope binding energy, resulting in $\mathrm{BBHs}$ 



Figure 3. Comoving merger rate $\left(\mathrm{Gpc}^{-3} \mathrm{yr}^{-1}\right)$ as a function of present day galaxy mass and metallicity (left) and lookback time to the formation of the progenitor (right). The distribution has been integrated over formation time (left) and progenitor metallicity (right). Side panels show the integrated rates for our total sample (solid lines) as well as the restricted GW150614-like sample (dotted lines).

that merge at later times. Except for the very low metallicity progenitors, we do not expect mergers from recently formed stars.

\section{FORMATION OF BBH MERGER CANDIDATES}

We now combine the number of low-metallicity stars formed in different galaxies at different epochs with the number of mergers after a certain delay time for different progenitor metallicities (see equation 1). We assume a binary fraction of 0.7 (Sana et al. 2012). Fig. 3 shows the merger rates as a function of host galaxy mass, progenitor formation time and metallicity. The distribution is bimodal with early formation of $Z_{\mathrm{p}} \gtrsim 0.1 \mathrm{Z}_{\odot}$ progenitors now present in massive galaxies and lower metallicity progenitors forming later in dwarf galaxies. The latter have limited star formation but are numerous and have a low metallicity. The contribution of dwarf galaxies is sensitive to the extrapolation of the low-mass galaxy SMF below observational completeness but the relatively flat galaxy mass distribution is robust to those uncertainties.

Integrated over all galaxy masses, the formation time of the progenitors is a rather flat distribution over the last $8 \mathrm{Gyr}$. We do not recover the strongly bimodal birth time distribution from Belczynski et al. (2016) because of our more accurate treatment of the star-forming gas metallicity and star formation. Most of the progenitors form around $Z_{\mathrm{p}} \simeq 0.1 \mathrm{Z}_{\odot}$. Many stars form at higher $Z_{\mathrm{p}}$, but the number of mergers per unit solar mass is drastically reduced. At lower progenitor metallicity, more systems merge, but the amount of stars formed is low. If we were to include recently proposed fast merger channels (Mandel \& de Mink 2016; Marchant et al. 2016), the distribution of host galaxies and formation times would be very similar to the distribution of low-metallicity stars, with a possible contribution from low-redshift galaxies.

The total merger rate we find is $\mathcal{R}=850 \mathrm{Gpc}^{-3} \mathrm{yr}^{-1}$ for our total mass sample and $\mathcal{R}=150 \mathrm{Gpc}^{-3} \mathrm{yr}^{-1}$ when we restrict ourselves to the exact masses observed in GW150914. After the first observing run, the LIGO estimate of the merger rate of GW150914-like black holes is 2-53 $\mathrm{Gpc}^{-3} \mathrm{yr}^{-1}$ (Abbott et al. 2016a). While our model is based on standard assumptions for galaxy evolution and massive binary evolution, the total predicted merger rate overestimates the observed rate only by a factor 3 . Choosing a metallicity calibration that predicts a lower MZR, a lower binary fraction and/or higher common envelope binding energy will naturally decrease these numbers. As we focus on formation conditions rather than absolute rates, we choose not to fine tune our model.

\section{DISCUSSION AND CONCLUSION}

In this Letter, we compute when and where GW150914 most likely formed. Using only the strong constraint on the progenitor's metallicity and combining a state of the art BPS model with a complete cosmological description for the evolution of low-metallicity gas, we find that GW150914 likely formed in a massive galaxy at $1 \leq z_{\mathrm{f}}$ $\leq 2$, but later formation in a dwarf galaxy is also possible. In fact the distribution of $\mathrm{BBH}$ merger progenitor formation times is remarkably flat for $t_{\mathrm{f}} \simeq 1-10 \mathrm{Gyr}$, and differs from the strongly bimodal distribution from Belczynski et al. (2016). Their computation is based on the metallicity evolution of DLAs, which ignores the crucial mass dependence of the metallicity. Our model also includes galactic mergers, which allow BBH progenitors formed in dwarf galaxies to end up in massive systems at $t_{\mathrm{m}}$ and strongly increase the amount of mergers in the latter. Still, we find a large contribution of mergers in dwarf galaxies, which is radically different from the distribution of present day stars, supernovae and $\mathrm{BH}$ which are strongly concentrated around $M_{\mathrm{gal}} \simeq 10^{11} \mathrm{M}_{\odot}$. This Letter presents the first determination of the formation conditions for the massive BBH mergers we currently observe. Without fine tuning, the total merger rate we predict is compatible with the LIGO detection rate. 
Our Letter assumes that the only environmental impact on stellar evolution is progenitor metallicity, allowing us to decouple galactic evolution and stellar evolution, including multiplicity and the IMF. As such, the large uncertainties in massive stellar evolution only affect our absolute merger rate, but not its dependence on galaxy mass and formation time. Unless the metallicity dependence of stellar evolution were to be drastically revised, our model can be easily rescaled for different models of massive stellar evolution.

Uncertainties also affect our model for galaxy evolution, especially in small galaxies at high redshifts where star formation rates and particularly metallicity are very hard to determine observationally. We have assumed that dwarf galaxies form the same amount of massive binaries per unit solar mass than larger galaxies, neglecting the fact that they may not host large enough molecular clouds to do so. As our understanding of high-redshift star formation and stellar evolution improves with data from the James Webb Space Telescope and the Wide Field Infrared Survey Telescope, our method will become a valuable tool to understand $\mathrm{BBH}$ mergers.

As Advanced LIGO and Advanced Virgo reach their design sensitivity (Abbott et al. 2016c), they will detect hundreds of $\mathrm{BBH}$ mergers, up to $z_{\mathrm{m}} \lesssim 1$. BBHs merging at these redshifts formed during the peak of cosmic star formation, with a rather flat distribution of galaxy mass. In this context, this will provide strong tests of our models and the otherwise elusive nature of high-redshift star formation and/or the metallicities of high-redshift or faint galaxies. Our method can further be combined with galaxy catalogues to predict typical distance distributions and sky localizations for future detections.

\section{ACKNOWLEDGEMENTS}

Support for AL and PFH was provided by an Alfred P. Sloan Research Fellowship, NASA ATP Grant NNX14AH35G, and NSF Collaborative Research Grant No. 1411920 and CAREER Grant No. 1455342. Support for SGK was provided by NASA through Einstein Postdoctoral Fellowship Grant No. PF5-160136 awarded by the Chandra X-ray Center, which is operated by the Smithsonian Astrophysical Observatory for NASA under contract NAS8-03060. DC was supported through the Walter Burke Institute for Theoretical Physics and the Sherman Fairchild Foundation and the Caltech Department of Astronomy. The authors thank Chris Pankow, Evan Kirby, Xiangcheng Ma, Fangzhou Jiang, and Peter Behroozi for very helpful and stimulating discussions. We thank the referee for a constructive report that improved and clarified the Letter.

\section{REFERENCES}

Abbott B. P. et al., 2016a, preprint (arXiv:1602.03842)

Abbott B. P. et al., 2016b, Phys. Rev. Lett., 116, 061102

Abbott B. P. et al., 2016c, Phys. Rev. Lett., 116, 131103

Abbott B. P. et al., 2016d, ApJ, 818, L22

Behroozi P. S., Wechsler R. H., Conroy C., 2013, ApJ, 770, 57

Belczynski K., Taam R. E., Kalogera V., Rasio F. A., Bulik T., 2007, ApJ, 662,504

Belczynski K., Kalogera V., Rasio F. A., Taam R. E., Zezas A., Bulik T., Maccarone T. J., Ivanova N., 2008, ApJS, 174, 223
Belczynski K., Bulik T., Fryer C. L., Ruiter A., Valsecchi F., Vink J. S., Hurley J. R., 2010, ApJ, 714, 1217

Belczynski K., Holz D. E., Bulik T., O’Shaughnessy R., 2016, Nature, 534, 512

Berg D. A., Skillman E. D., Garnett D. R., Croxall K. V., Marble A. R., Smith J. D., Gordon K., Kennicutt R. C., Jr, 2013, ApJ, 775, 128

Cole S., Lacey C. G., Baugh C. M., Frenk C. S., 2000, MNRAS, 319, 168

Cole S., Helly J., Frenk C. S., Parkinson H., 2008, MNRAS, 383, 546

Dominik M., Belczynski K., Fryer C., Holz D. E., Berti E., Bulik T., Mandel I., O'Shaughnessy R., 2013, ApJ, 779, 72

Downing J. M. B., Benacquista M. J., Giersz M., Spurzem R., 2011, MNRAS, 416, 133

Dvorkin I., Silk J., Vangioni E., Petitjean P., Olive K. A., 2015, MNRAS, 452, L36

Dvorkin I., Vangioni E., Silk J., Uzan J.-P., Olive K. A., 2016, MNRAS, 461, 3877

Erb D. K., Steidel C. C., Shapley A. E., Pettini M., Reddy N. A., Adelberger K. L., 2006, ApJ, 647, 128

Fakhouri O., Ma C.-P., 2008, MNRAS, 386, 577

Guo Q., White S. D. M., 2008, MNRAS, 384, 2

Hartwig T., Volonteri M., Bromm V., Klessen R. S., Barausse E., Magg M., Stacy A., 2016, MNRAS, 460, L74

Henry R. B. C., Kwitter K. B., Jaskot A. E., Balick B., Morrison M. A., Milingo J. B., 2010, ApJ, 724, 748

Hopkins P. F., Kereš D., Oñorbe J., Faucher-Giguère C.-A., Quataert E., Murray N., Bullock J. S., 2014, MNRAS, 445, 581

Hurley J. R., Tout C. A., Pols O. R., 2002, MNRAS, 329, 897

Ivanova N., Taam R. E., 2004, ApJ, 601, 1058

Kewley L. J., Ellison S. L., 2008, ApJ, 681, 1183

Kinugawa T., Inayoshi K., Hotokezaka K., Nakauchi D., Nakamura T., 2014, MNRAS, 442, 2963

Kobulnicky H. A., Kewley L. J., 2004, ApJ, 617, 240

Kroupa P., 2001, MNRAS, 322, 231

Lacey C., Cole S., 1993, MNRAS, 262, 627

Lackner C. N., Cen R., Ostriker J. P., Joung M. R., 2012, MNRAS, 425, 641

Lee H., Skillman E. D., Cannon J. M., Jackson D. C., Gehrz R. D., Polomski E. F., Woodward C. E., 2006, ApJ, 647, 970

Lipunov V. M., Postnov K. A., Prokhorov M. E., 1997, MNRAS, 288, 245

Ma X., Hopkins P. F., Faucher-Giguère C.-A., Zolman N., Muratov A. L., Kereš D., Quataert E., 2016, MNRAS, 456, 2140

Maller A. H., Katz N., Kereš D., Davé R., Weinberg D. H., 2006, ApJ, 647, 763

Mandel I., de Mink S. E., 2016, MNRAS, 458, 2634

Mannucci F. et al., 2009, MNRAS, 398, 1915

Marchant P., Langer N., Podsiadlowski P., Tauris T. M., Moriya T. J., 2016, A\&A, 588, A50

O’Shaughnessy R., Kalogera V., Belczynski K., 2010, ApJ, 716, 615

Pettini M., Pagel B. E. J., 2004, MNRAS, 348, L59

Rafelski M., Wolfe A. M., Prochaska J. X., Neeleman M., Mendez A. J., 2012, ApJ, 755, 89

Rodriguez C. L., Morscher M., Pattabiraman B., Chatterjee S., Haster C.-J., Rasio F. A., 2015, Phys. Rev. Lett., 115, 051101

Sana H. et al., 2012, Science, 337, 444

Sipior M. S., Sigurdsson S., 2002, ApJ, 572, 962

Steidel C. C. et al., 2014, ApJ, 795, 165

Tomczak A. R. et al., 2014, ApJ, 783, 85

Tremonti C. A. et al., 2004, ApJ, 613, 898

This paper has been typeset from a $\mathrm{T}_{\mathrm{E}} \mathrm{X} / \mathrm{L} \mathrm{A} \mathrm{E} \mathrm{X}$ file prepared by the author. 